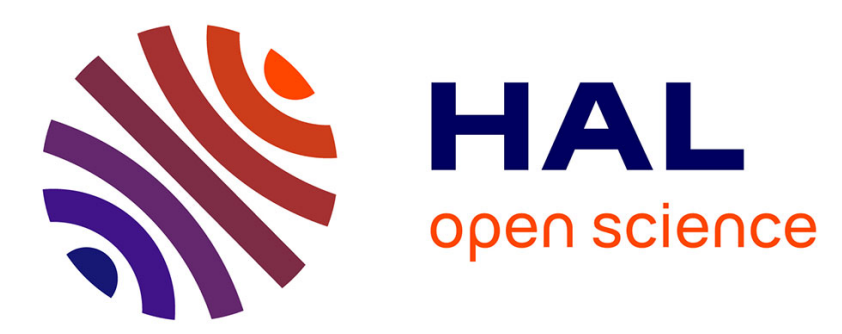

\title{
A self-consistent analytical model for the upstream magnetic-field and ion-beam properties in Weibel-mediated collisionless shocks
}

\author{
C. Ruyer, L. Gremillet, G. Bonnaud, C. Riconda
}

\section{- To cite this version:}

C. Ruyer, L. Gremillet, G. Bonnaud, C. Riconda. A self-consistent analytical model for the upstream magnetic-field and ion-beam properties in Weibel-mediated collisionless shocks. Physics of Plasmas, 2017, 24 (4), pp.41409. 10.1063/1.4979187 . hal-01542835

\section{HAL Id: hal-01542835 \\ https://hal.sorbonne-universite.fr/hal-01542835}

Submitted on 20 Jun 2017

HAL is a multi-disciplinary open access archive for the deposit and dissemination of scientific research documents, whether they are published or not. The documents may come from teaching and research institutions in France or abroad, or from public or private research centers.
L'archive ouverte pluridisciplinaire HAL, est destinée au dépôt et à la diffusion de documents scientifiques de niveau recherche, publiés ou non, émanant des établissements d'enseignement et de recherche français ou étrangers, des laboratoires publics ou privés. 


\section{A self-consistent analytical model for the upstream magnetic-field and ion-beam properties in Weibel-mediated collisionless shocks}

C. Ruyer, L. Gremillet, G. Bonnaud, and C. Riconda

Citation: Physics of Plasmas 24, 041409 (2017); doi: 10.1063/1.4979187

View online: http://dx.doi.org/10.1063/1.4979187

View Table of Contents: http://aip.scitation.org/toc/php/24/4

Published by the American Institute of Physics

\section{Articles you may be interested in}

On the generation of magnetized collisionless shocks in the large plasma device

Physics of Plasmas 24, 041405 (2017); 10.1063/1.4978882

Magnetic field production via the Weibel instability in interpenetrating plasma flows

Physics of Plasmas 24, 041410 (2017); 10.1063/1.4982044

Numerical modeling of laser-driven experiments aiming to demonstrate magnetic field amplification via turbulent dynamo

Physics of Plasmas 24, 041404 (2017); 10.1063/1.4978628

Particle acceleration in laser-driven magnetic reconnection

Physics of Plasmas 24, 041408 (2017); 10.1063/1.4978627

Formation of high-speed electron jets as the evidence for magnetic reconnection in laser-produced plasma Physics of Plasmas 24, 041406 (2017); 10.1063/1.4978883

Electron holes in phase space: What they are and why they matter

Physics of Plasmas 24, 055601 (2017); 10.1063/1.4976854

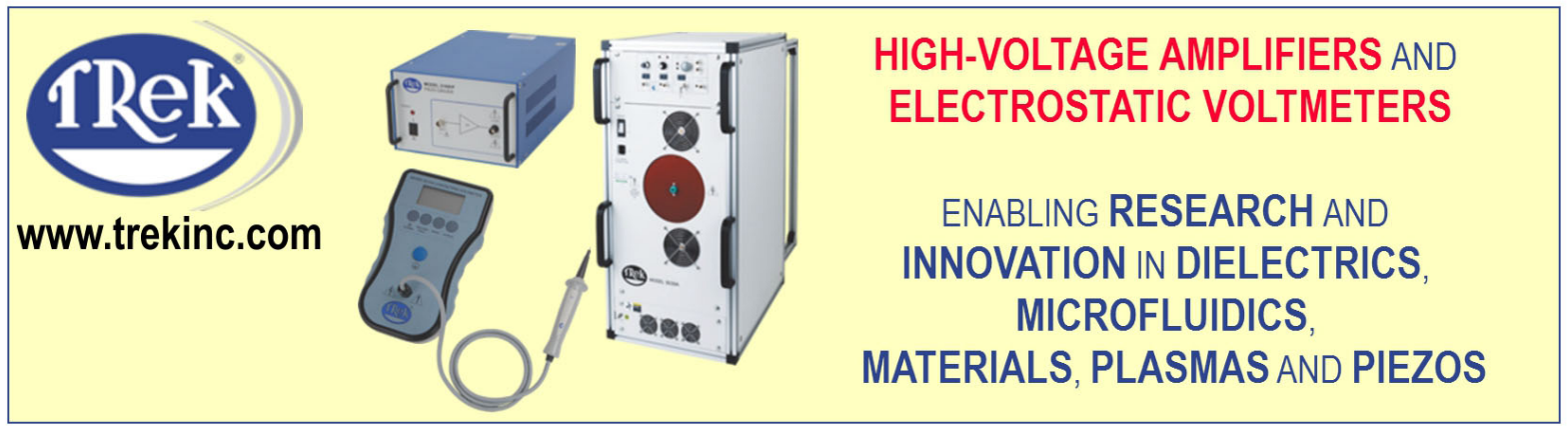




\title{
A self-consistent analytical model for the upstream magnetic-field and ion-beam properties in Weibel-mediated collisionless shocks
}

\author{
C. Ruyer, ${ }^{1,2,3, a)}$ L. Gremillet, ${ }^{1, b)}$ G. Bonnaud, ${ }^{4}$ and C. Riconda ${ }^{5}$ \\ ${ }^{1}$ CEA, DAM, DIF, F-91297 Arpajon, France \\ ${ }^{2}$ LULI, Ecole Polytechnique-CNRS-CEA-UPMC, 91128 Palaiseau, France \\ ${ }^{3}$ High Energy Density Science Division, SLAC National Accelerator Laboratory, Menlo Park, \\ California 94025, USA \\ ${ }^{4}$ CEA, Saclay, INSTN, F-91191 Gif-sur-Yvette, France \\ ${ }^{5}$ LULI, Sorbonne Universités-UPMC-Ecole Polytechnique-CNRS-CEA, 75005 Paris, France
}

(Received 15 October 2016; accepted 17 February 2017; published online 7 April 2017)

\begin{abstract}
A theoretical and numerical analysis is carried out for turbulent collisionless shocks mediated by the ion-Weibel instability during high-velocity plasma collisions. We develop a simple model based on the coalescence dynamics of the ion current filaments, which predicts the spatio-temporal evolution of the magnetic fluctuations formed in the upstream plasma region. From comparison with particle-in-cell simulations, our model is shown to correctly capture the magnetic-field and ion-beam properties during the early-time shock propagation. Published by AIP Publishing.

[http://dx.doi.org/10.1063/1.4979187]
\end{abstract}

\section{INTRODUCTION}

Fully kinetic modeling of collisionless turbulent shocks is now accessible to particle-in-cell (PIC) simulations using modern supercomputers, hence providing a first-principles description of various high-energy astrophysical scenarios. ${ }^{1-4}$ According to an increasing body of theoretical research ${ }^{5-8}$ such shocks likely originate from the microinstabilities excited in counterstreaming plasmas. The Fermitype acceleration process taking place in the electromagnetic turbulence induced around the shock's discontinuity is held responsible for the generation of suprathermal particles and radiations in powerful astrophysical objects (e.g., supernova remnants, gamma ray bursts, or active galactic nuclei). ${ }^{9-11}$

During the collision of two electron-ion plasma flows, electron-driven micro-instabilities ${ }^{12}$ first develop and saturate, causing fast electron isotropization over the overlap region. In the absence of an external magnetic field and for high-velocity $\left(v_{i} \gtrsim 10^{-3} c\right.$, where $c$ is the speed of light in vacuum), high-Mach-number $\left(M_{s} \gg 1\right)$ flows, the ion Weibel-filamentation instability subsequently drives the electromagnetic turbulence, ${ }^{7}$ which progressively scatters the interacting ions until complete isotropization is reached.

In recent years, increasing experimental efforts have been devoted to this topic using plasma plumes produced by high-power lasers. ${ }^{13-16}$ The generation of Weibel-unstable, counterstreaming ablative flows is a challenging experimental task, which requires multi-kilojoule laser systems and sophisticated diagnostic techniques such as proton-radiography. ${ }^{17,18}$ The first proton-radiograph evidencing selfinduced, kinetic-scale magnetic filaments was reported in Ref. 19, yet one had to wait for Ref. 14 to confront similar measurements with PIC simulations, indicating that Weibeltype instabilities were likely responsible for the observed

\footnotetext{
a) charles.ruyer@cea.fr

b)laurent.gremillet@cea.fr
}

structures. However, achieving truly collisionless plasma conditions of astrophysical relevance puts strong constraints on the required plasma density and temperature. ${ }^{20}$ In this regard, Refs. 16 and 21 presented a thorough analysis of the plasma conditions attained during the two-stream interaction, alongside a quantitative interpretation of the data by a fully three-dimensional PIC simulation. Although proof was made that the observed filamentary structures did result from the ion-Weibel instability, none of these experiments succeeded in creating a fully formed, propagating shock. The reason is that the effective interaction length in the streamwise direction was insufficient to isotropize the incoming ions.

In this novel field of laboratory astrophysics, experimental progress is linked to a more accurate characterization of the turbulent fields and plasma properties and, also, to a better understanding of the Weibel-mediated regimes accessible on presently available laser systems. In this respect, it should be stressed that, despite the use of state-of-the-art supercomputers, the PIC simulations performed so far to help design or interpret the experiments have resorted to strong numerical simplifications such as reduced ion-to-electron mass ratios. Hence, the knowledge of the exact conditions required for laser-driven shock formation remains an open issue, which strongly motivates further theoretical work.

Once isotropized, the beam particles are expected to accumulate in the center of the system until the standard Rankine-Hugoniot (RH) jump conditions are satisfied, similarly to what occurs in a hydrodynamic shock. ${ }^{22}$ These conditions, which express conservation of particle number, momentum, and energy across the shock front region, give the well-known plasma density jump across a strong shock: $n_{d} / n_{u}=\left(\Gamma_{\mathrm{ad}}+1\right) /\left(\Gamma_{\mathrm{ad}}-1\right)$, where the subscripts " $u$ " and " $d$ " denote upstream and downstream quantities, respectively. For a non-relativistic 3D (resp. 2D) system, the adiabatic index $\Gamma_{\mathrm{ad}}$ is equal to $5 / 3$ (resp. 2), leading to a compression factor of 4 (resp. 3). Therefore, following particle isotropization, each beam should undergo a density 
increase of $100 \%$ in 3D (50\% in 2D) in the accumulation (downstream) region before the shock is formed and starts propagating.

Theoretical estimates of the isotropization and shock formation times, supported by PIC simulations, were given by Bret et al. ${ }^{23,24}$ for colliding relativistic pair plasmas. In this case, the magnetic fluctuations reached at the end of the linear Weibel instability phase are strong enough to fully scatter the interacting particle over typically one bouncing period inside the magnetic filaments. Hence, the RH conditions are fulfilled after a sufficient number of particles have piled up in the central region. ${ }^{24}$

By contrast, for experimentally relevant electron-ion plasmas, the Weibel instability saturates well before the time of ion isotropization. In a previous paper, ${ }^{25}$ we demonstrated that the post-saturation evolution of the instability proceeds through mergers of ion current filaments. We developed an analytical model combining approximate quasilinear relations and a simple description of the coalescence of electronscreened ion micro-currents. This model was successfully compared with periodic PIC simulations in the early nonlinear instability stage for symmetric counterpropagating beams (i.e., of identical densities, absolute velocities, temperatures, masses, and charges). However, at a later stage of the plasma collision, the two interacting beams may present locally different properties, especially at the edges of the overlap region. As the overlap region continues to thicken, the assumption of locally symmetric beams made in Ref. 25 becomes less and less valid. In a follow-up paper, ${ }^{26}$ the locally asymmetric character of the system (which consists of a relatively cold ion beam interacting with a hotter ion population in a background of isotropized, screening electrons) was shown to slow down the merging dynamics of the current filaments, thus leading to a scaling of the ion isotropization length $\left(L_{\text {iso }} \propto m_{i}^{0.9}\right)$ significantly larger than previously assumed $\left(L_{\text {iso }} \sim 100 \mathrm{c} / \omega_{p i} \propto m_{i}^{1 / 2}\right)$. ${ }^{1}$ Interestingly, our estimates are also larger than those proposed in Ref. 27 for relativistic to ultra-relativistic electron-ion plasma collisions, $L_{\text {iso }} \propto \log \left(m_{i} / m_{e}\right) c / \omega_{p i}$. Note that electron screening effects were neglected in this study when modeling the merging dynamics of the ion micro-currents.

The non-relativistic results of Refs. 25 and 26 were obtained in the context of a forming shock, and thus may be useful to design future plasma collision experiments. Yet, for the astrophysical scenarios of main interest, the processes at stake during shock propagation (e.g., the generation of highenergy particles and radiation) are of greater importance, so that the shock should be considered to be formed. In a quasistationary propagation regime, the turbulence is sustained in the upstream region by the interaction of the incoming ions with a counterstreaming, hot ion population issued from the downstream or the shock front. ${ }^{1,2,28}$ As in the shock formation phase, this turbulent region should be strong/long enough to dissipate the incoming flow energy into thermal energy.

In this paper, we address the shock structure during its early-time propagation phase, through the analysis of the Weibel-filamentation instability taking place in the vicinity of the shock front. Here "early-time propagation" means that the shock wave fulfills the RH conditions, yet it is not selfsustained by the sole reflected particles (i.e., the system is still influenced by the particles transmitted through the contact discontinuity region). The main plasma features are studied in Sec. II by means of PIC simulations and quantitative estimates for the different particle populations at play. The analytical model worked out in Ref. 26 for the shock formation stage is detailed in Sec. III. In Sec. IV, this model is applied to the early-time shock propagation stage, yielding predictions of the spatial field and plasma profiles. Finally, Sec. V gathers our conclusions and prospects.

\section{FORMATION AND PROPAGATION OF A WEIBEL-MEDIATED COLLISIONLESS SHOCK}

\section{A. Formation of the downstream}

We have simulated the collective interaction between two symmetric, counterstreaming plasmas using the PIC code CALDER. ${ }^{29}$ In all of the simulations, the electron-ion beams are initially placed on both sides of the domain's center and are characterized by Maxwellian distributions of temperatures $T_{e}=T_{i}=m_{e} c^{2} / 100$ and $\left(x\right.$-aligned) drift velocities $v_{e}=v_{i}$ $= \pm v_{0}$ with $v_{0} \in(0.2 c, 0.4 c)$. The initial density of each beam is $n_{0}=n_{i, e} / 2 \equiv 0.5$ and the ion charge is $Z_{i}=1$. Depending on the ion-to-electron mass ratio $m_{i} / m_{e}$ (where the subscripts "e" and " $i$ " denote electron and ion quantities), the size of the simulated domain is $L_{x} \times L_{y}=8400 \times 538\left(c / \omega_{p e}\right)^{2}$ for $m_{i} / m_{e}$ $=25, L_{x} \times L_{y}=12600 \times 538\left(c / \omega_{p e}\right)^{2}$ for $m_{i} / m_{e}=100$, and $L_{x} \times L_{y}=22627 \times 1131\left(c / \omega_{p e}\right)^{2}$ for $m_{i} / m_{e}=400$. We have introduced $\omega_{p s}=\sqrt{n_{s} q_{s}^{2} / m_{s} \epsilon_{0}}$, the plasma frequency of the sth species, where $\epsilon_{0}, n_{s}, q_{s}$, and $m_{s}$ stand for the permittivity of vacuum, density, charge, and mass of the sth species. The space and time steps are $\Delta x=\Delta y=0.3 c / \omega_{p e}$ and $\Delta t$ $=0.2 \omega_{p e}^{-1}$, respectively. Care has been taken to verify that a smaller mesh size does not quantitatively affect the simulation results. Each cell contains initially 10 macroparticles per beam species. For both particles and fields, absorbing (resp. periodic) boundary conditions are used in the longitudinal, $x$ (resp. transverse, $y$ ) direction.

Note that our choice of drift velocities, $v_{0}=0.2-0.4 c$, and mass ratios, $m_{i} / m_{e} \leq 400$, is the result of a tradeoff between numerical feasibility and our focus on nonrelativistic shocks relevant to supernova remnant scenarios and current laser experiments (i.e., characterized by $m_{i} / m_{e}$ $\sim 2000$ and $v_{0} \sim 0.01 c$ ). The computational load of our $2 \mathrm{D}$ simulations indeed scales as $L_{t} \times L_{x} \times L_{y}$ (the space-time simulation domain). Taking $L_{x} \sim v_{0} L_{t} \sim L_{\text {iso }}$ and $L_{y} \propto c / \omega_{p i}$ and using Eq. (30) below leads to a computational cost varying as $\sim\left(m_{i} / m_{e}\right)^{2.3} / v_{0}$. Keeping it at an acceptable level therefore requires us to artificially reduce $m_{i} / m_{e}$ and increase $v_{0}$ (yet keeping it significantly lower than $c$ ) relative to the actual physical values.

Figures 1(a)-1(d) display simulation results obtained for $m_{i} / m_{e}=25$ and $v_{0}=0.4 \mathrm{c}$. All the panels correspond to the same time $t \omega_{p e}=4830$, i.e., after shock formation. The density map plotted in Fig. 1(a) evidences a shocked (downstream) region centered around $x=4200 c / \omega_{p e}$ and of thickness $\sim 1500 \mathrm{c} / \omega_{p e}$. Its density profile is essentially 
(a) Ion density $n_{i} / n_{0}$

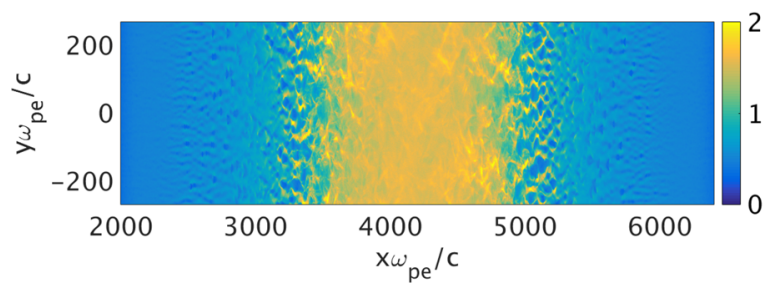

(c) Transverse temperatures

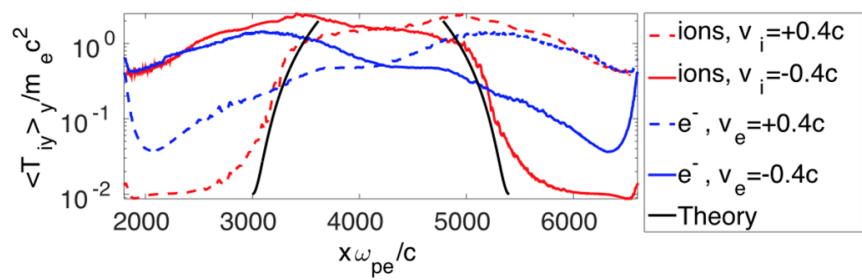

(b) Magnetic field $e B_{z} / m_{e} \omega_{p e}$

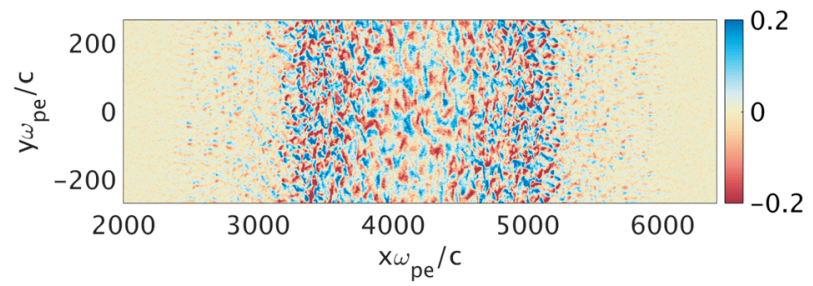

(d) Anisotropy ratio

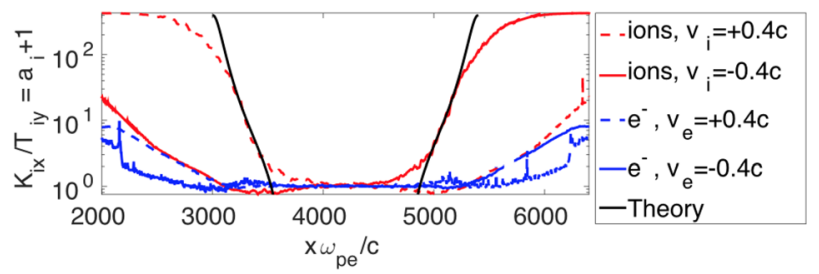

FIG. 1. Collisionless shock simulation with $m_{i}=25 m_{e}, v_{e, i}= \pm 0.4 c$ and $T_{e, i}^{(0)}=0.01 m_{e} c^{2}$ and $n_{e, i}=0.5$. (a) Total ion density. (b) Magnetic field $e B_{z} / m_{e} \omega_{p e}$. (c) Transversely averaged ion (red) and electron (blue) temperatures $\left(T_{i, e v} / m_{e} c^{2}\right)$. (d) Transversely averaged anisotropy factor $\left(a_{s}+1 \equiv K_{s x} / T_{s y}\right)$ for the ions (red) and electrons (blue). In (c) and (d), the dashed/solid lines distinguish between beams of initial (far upstream) positive/negative mean velocities, except for the black solid lines, which plot the ion temperature and anisotropy predicted by Eqs. (25) and (26), respectively. All maps and curves are extracted at time $t \omega_{p e}=4830$.

uniform, corresponding to a compression factor of $\sim 1.5 /$ 0.5-3 with respect to the upstream plasma, in agreement with the RH conditions.

Figure 1(a) reveals the growth of density filaments in the shock transition (precursor) regions $\left(2500 \leqq x \omega_{p e} / c \leqq 3000\right.$ and $\left.5500 \leqq x \omega_{p e} / c \lesssim 6000\right)$. These density modulations are correlated with the Weibel-driven magnetic fluctuations seen in Fig. 1(b). The instability is triggered by the incoming cold ions $\left(v_{i} \sim v_{0}\right)$ counterstreaming against a population of hotter ions $\left(T_{i y} \simeq m_{e} c^{2},-0.5 c \lesssim v_{i} \lesssim-0.4 c\right)$ originating from the shocked region, as shown in the ion phase space $\left(x-p_{x}\right)$ of Fig. 2(a). The latter population comprises ions that have either leaked out from the downstream or been reflected at the shock front. Except for those that have been transmitted essentially ballistically early in the interaction, these ions have traveled across a longer turbulent region than the incoming ions, and so present a broader thermal spread. The strong density modulations seen in Fig. 1(a) are due to the cold incoming ions which are more easily pinched by the magnetic fluctuations than the hotter counterstreaming ions. As is well known, ${ }^{28,30}$ the magnetic filaments formed in the transition region evolve into more isotropic, clumpy structures in the downstream.

The process of ion isotropization across the magnetically turbulent shock front is illustrated in Fig. 1(d), where (a) $x-p_{x}$ ion phase space

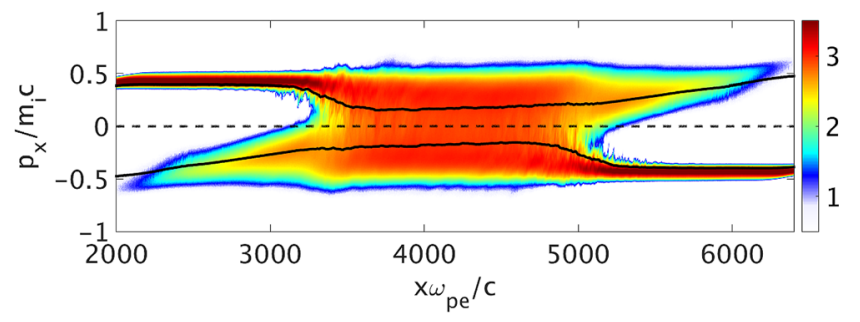

(c) $\Gamma / \omega_{p e}$

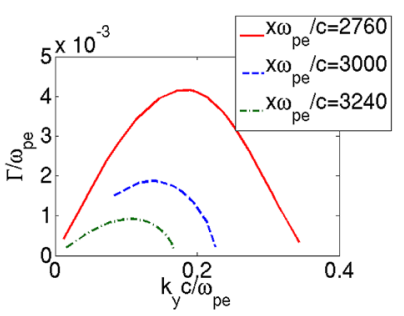

(d) $\left\langle n v_{x}\right\rangle$ at $x \omega_{p e} / c=2760$

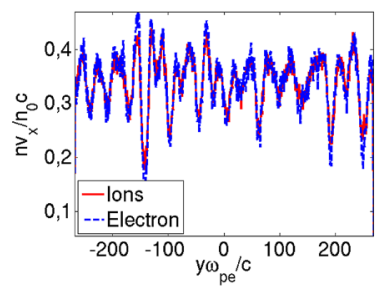

(b) $\log \left[\mid \mathrm{FFT}_{y}\left(B_{z}\left(m_{e} \omega_{p e} / e\right) \mid\right]\right.$

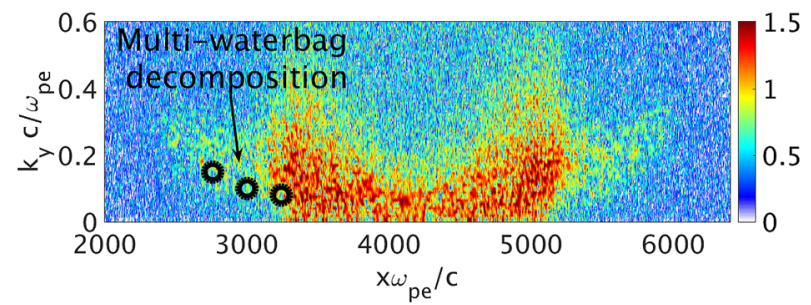

(e) $\left\langle n v_{x}\right\rangle$ at $x \omega_{p e} / c=3000$

(f) $\left\langle n v_{x}\right\rangle$ at $x \omega_{p e} / c=3240$

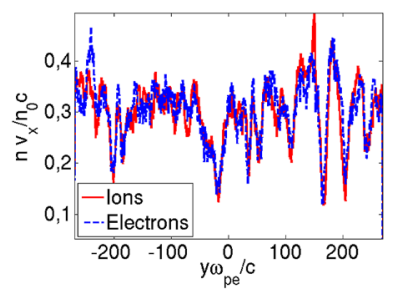

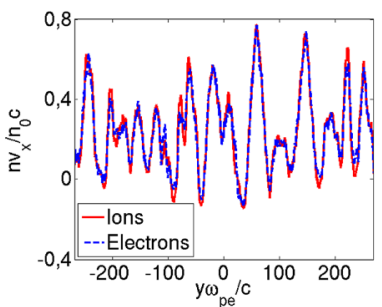

FIG. 2. (a) $x-p_{x}$ ion phase space ( $x$ is in units of $c / \omega_{p e}$, and $p_{x}$ in units of $m_{i} c$ ) at $t \omega_{p e}=4830$ (in log scale). The simulation parameters are $m_{i}=25 m_{e}, T_{i, e}^{(0)}=0.01 m_{e} c^{2}$, and $v_{i, e}= \pm 0.4 c$. The ( $y$-averaged) mean ion velocity $v_{i} / c$ of each beam and the total normalized current $j_{x} / q_{e} n_{0} c$ are superimposed as solid and dashed black lines, respectively. (b) Transverse Fourier transform of the magnetic field $\left|B_{z}\left(x, k_{y}\right)\right|$ at $t \omega_{p e}=4830$. (c) Weibel growth rate calculated at $x \omega_{p e} / c=2760$ (red plain line), 3000 (blue dashed line), and 3240 (green dotted-dashed line) using a multi-waterbag decomposition ${ }^{31}$ of the $(y$ averaged) local distribution functions. The wave numbers maximizing the growth rates of (c) are superposed in (b) as black circles. (d)-(f) Lineouts of the mean ion (solid red line) and electron (dashed blue line) flux densities $n v_{x} / n_{0} c$ at $x \omega_{p e} / c=2760$ (d), $x \omega_{p e} / c=3000$ (e), and $x \omega_{p e} / c=3240$ (f) at $t \omega_{p e}=4830$. 
the ratio between the (transversely averaged) longitudinal $\left(K_{i x}=m_{i} v_{i}^{2}+T_{i x}\right)$ and transverse $\left(T_{i y}\right)$ momentum fluxes is plotted as a function of the longitudinal position. As expected, this ratio drops down to unity in the downstream.

Assuming that the downstream particles are fully isotropized (at least in the vicinity of the contact plane), the ion temperature $T_{i, d}$ can be estimated from energy conservation. Equating the mean energy of the downstream ions $\left(3 T_{i, d} / 2\right.$ in 3D and $T_{i, d}$ in 2D) with the initial kinetic ion energy $m_{i} v_{0}^{2} / 2$ (assuming an initially cold flow), we obtain $T_{i, d} \simeq m_{i} v_{0}^{2} / 2$ in $2 \mathrm{D}$ and $T_{i, d} \simeq m_{i} v_{0}^{2} / 3$ in $3 \mathrm{D}$. This estimate is supported by the ion temperature profiles displayed in Fig. 1(c) (solid and dashed red lines): in the downstream, one finds $T_{i, d} \simeq 2 m_{e} c^{2}$, as predicted by the above formula. Note that the artifacts visible on the edges of the electron temperature [Fig. 1(c)] and anisotropy [Fig. 1(d)] profiles are due to the fastest electrons at the beam heads crossing the tails of the counterstreaming beams, thus leaking out into vacuum before being reflected back by charge separation fields. This spurious effect, however, occurs at the outer edges of the ion-ion overlap region, and does not affect the nonlinear ion-Weibel stage under scrutiny.

From the RH conditions, ${ }^{22}$ the shock is expected to move at a constant velocity $v_{\mathrm{sh}}=v_{0} / 2\left(\right.$ resp. $\left.v_{0} / 3\right)$ in $2 \mathrm{D}$ (resp. 3D), consistent with the simulation. Introducing the quasi-isotropisation time $t_{\text {iso }}$ (estimated below), the time required for the shock front to reach a position $x$ from the center $\left(x_{0}\right)$ of the overlap region is

$$
t_{\text {front }}(x)=t_{\text {iso }}+\frac{\left|x-x_{0}\right|}{v_{\text {sh }}} .
$$

\section{B. Asymmetric ion-ion interaction in the upstream region}

In the $x-p_{x}$ ion phase space displayed in Fig. 2(a), the mean velocities of the colliding ions beams are superimposed as solid black curves. Interestingly, the mean velocity of each beam does not vanish in the downstream, which shows that, at the time under consideration $\left(t \omega_{p e}=4830\right)$, the incoming ions are still being transmitted though the shock front and the downstream, and are leaking into the upstream at the opposite side. Recall that for a fully formed collisionless shock, one expects a downstream region thick enough that the incoming ions cannot be directly transmitted upstream. However, in the present case of initially symmetric counterstreaming beams (of identical densities, absolute velocities, temperatures, masses, and charges), we do not expect a significant difference between the reflected (or leaking) and transmitted ion populations, and so the shocks properties revealed by Figs. 1 and 2 should resemble those of a fully formed, self-sustained shock (i.e., no longer influenced by its initial conditions).

The absolute mean velocity of the ions transmitted into the upstream is close (yet not equal) to that of the incoming ions, leading to a non-vanishing net ion current in the upstream. This net ion current, however, is efficiently neutralized on average (after integration along the $y$-direction) by the electrons, yielding an essentially null total current [black dashed line in Fig. 2(a)]. The upstream region then consists of two asymmetric (with respect to the density and temperature) counterstreaming ion beams, currentneutralized by a mostly isotropic electron background. This configuration triggers the ion Weibel-filamentation instability responsible for the density and magnetic modulations (characterized by a mostly perpendicular wave number) visible in Figs. 1(a) and 1(b).

The main difference with the system studied in Ref. 25 concerns the asymmetric character of the colliding beams in the upstream. In a frame comoving with the shock front, the incoming ion flow, initially of low temperature, is increasingly heated as it interacts with the magnetic fluctuations and moves closer to the shock front. By contrast, the counterstreaming ions are hot $\left(T_{h} \sim m_{e} c^{2}\right)$ and cool down when escaping from the turbulent region, as illustrated in Fig. 1(c). This asymmetric configuration affects the dynamics of the instability compared to what occurs in a symmetric twostream system. The electron and ion contributions to the current modulations can be assessed at different positions in Figs. 2(d)-2(f). The ion current modulations reach slightly higher amplitudes than the electron current close to the shock front $\left(x \omega_{p e} / c=3240\right)$, while the electron current modulations slightly prevail deeper in the upstream $\left(x \omega_{p e} / c=2760\right)$. Overall, however, there is no clear evidence for dominant electron or ion contributions in this spatial region.

These observations can be confronted to the results of an instability analysis performed at various positions in the upstream. For that purpose, we have decomposed the local $(y$-averaged $)$ electron and ion momentum distribution functions as a sum of waterbags using the method exposed in Ref. 31. Figure 2(c) plots the Weibel growth rates obtained from the fitted plasma distributions (using a total number of 2000 waterbags) at time $t \omega_{p e}=4830$ and positions $x \omega_{p e} /$ $c=2760,3000$, and 3240. As one moves closer to the shock front, the peak growth rate decreases from $\Gamma \simeq 4 \times 10^{-3} \omega_{p e}$ to $\simeq 9 \times 10^{-4} \omega_{p e}$, and shifts to lower wave numbers (from $\simeq 0.19 \omega_{p e} / c$ to $\left.\simeq 0.1 \omega_{p e} / c\right)$. This stabilization results from the increasingly hot and isotropic particle distributions. We have checked that the counterstreaming reflected/transmitted hot ion population is critical in destabilizing the system: neglecting its contribution in the Weibel dispersion relation does suppress the instability. Also, Fig. 2(a) shows that the spatial variation of the most unstable transverse $\left(k_{y}\right)$ wave number as predicted from linear theory correctly matches the simulated magnetic spectrum $B_{z}\left(x, k_{y}\right)$. In the following, we shall develop an analytical model for the Weibel-instabilitydriven evolution of the field and plasma properties.

\section{COLLECTIVE FILAMENT DYNAMICS IN THE UPSTREAM REGION}

\section{A. Characterization of the magnetic spectrum}

As shown above, the Weibel instability taking place upstream of the shock is triggered by the interaction of a cold (incoming, $c$-subscript) ion beam and a hot (transmitted/reflected, $h$-subscript) ion population, both immersed within a hot electron background (e-subscript). We will 
assume that each beams obeys a bi-Maxwellian distribution (normalized to unity) defined as

$$
f_{s}^{(0)}=\frac{m_{s}}{2 \pi \sqrt{T_{s x} T_{s y}}} \exp \left(-\frac{m_{s}\left(v_{x}-v_{s}\right)^{2}}{2 T_{s x}}-\frac{m_{s} v_{y}^{2}}{2 T_{s y}}\right) .
$$

In the above equation, the temperatures and drift velocities of the various ion and electron populations vary with the distance to the shock front. Yet, it will be assumed that the plasma evolution is smooth enough so that the following general electromagnetic dispersion relation still holds locally:

$$
\left(\omega^{2} \epsilon_{x x}-k_{y}^{2} c^{2}\right)\left(\omega^{2} \epsilon_{y y}-k_{x}^{2} c^{2}\right)-\left(\omega^{2} \epsilon_{x y}-k_{x} k_{y} c^{2}\right)^{2}=0 .
$$

The elements of the non-relativistic dielectric tensor $\epsilon_{\alpha \beta}$ involve the plasma dispersion function $\mathcal{Z}^{32}$ as detailed in Ref. 25. For a purely transverse $\left(k=k_{y}\right)$, non-propagating $(\Re(\omega)=0)$ Weibel eigenmode, the argument, $\xi$, of the function $\mathcal{Z}$ reads

$$
\xi_{s}=\frac{i \Gamma_{k_{y}}}{k_{y}} \sqrt{\frac{m_{s}}{2 T_{s y}}}
$$

where $\Gamma_{k_{y}} \equiv \Im \omega$ is the growth rate. Typical values of $\xi_{s}$ can be estimated from the growth rate curves of Fig. 2(c): $\left|\xi_{c}\right| \simeq 0.46<1$ and $\left|\xi_{h}\right| \simeq 0.13<1$ for the cold and hot ion populations, respectively. For the electrons, we have $\left|\xi_{e}\right|$ $\simeq 2.6 \times 10^{-3} \ll 1$. We will now proceed to simplify the linear dispersion relation to leading order in $\xi_{s}$ to obtain an estimate of the dominant magnetic wave number.

For two (hot and cold) ion populations immersed within a hot isotropic electron background (neutralizing the net ion current), one should solve, in principle, the full electromagnetic dispersion relation of Eq. (3). Yet this is made difficult by the non-vanishing $\epsilon_{x y}$ term in the asymmetric interaction region, which writes 25

$$
\epsilon_{x y}=\sum_{s} \frac{\omega_{p s}^{2}}{\omega^{2}} \frac{2 v_{s}}{v_{t h, s}}\left[\xi_{s}+\xi_{s}^{2} \mathcal{Z}\left(\xi_{s}\right)\right]
$$

where $v_{s}$ and $v_{t h, s}=\sqrt{2 T_{s y} / m_{s}}$ are the mean and thermal velocities of the sth species. From the above equation, we find $\epsilon_{x y} \propto \sum_{s} \omega_{p s}^{2} v_{s} / v_{t h, s}^{2}$ to leading order in $\xi_{s}$. It therefore proves convenient to solve the non-relativistic transverse dispersion relation in the frame $\mathcal{R}^{\prime}$ drifting at

$$
v_{f}=\frac{\frac{Z_{c}^{2} n_{c} v_{c}}{T_{c y}}+\frac{Z_{h}^{2} n_{h} v_{h}}{T_{h y}}+\frac{n_{e} v_{e}}{T_{e y}}}{\frac{Z_{c}^{2} n_{c}}{T_{c y}}+\frac{Z_{h}^{2} n_{h}}{T_{h y}}+\frac{n_{e}}{T_{e y}}} .
$$

In the frame $\mathcal{R}^{\prime}, \epsilon_{x y}$ vanishes to first order in $\xi_{s}$, and hence the Weibel dispersion relation simplifies to $\omega^{2} \epsilon_{x x}-k_{y}^{2}=0$. Consequently, to leading order in $\xi_{s}$, the Weibelfilamentation growth rate obeys an equation similar to that obtained in the symmetric case of Ref. 25. The general formula reads

$$
\Gamma_{k_{y}} \simeq \frac{k_{y}}{\sqrt{\pi}} \frac{\sum_{s} \omega_{p s}^{2} a_{s}^{\prime}-k_{y}^{2} c^{2}}{\sum_{s} \omega_{p s}^{2} \frac{a_{s}^{\prime}+1}{v_{t h, s}}},
$$

where the sum runs over $s \equiv[c, h, e]$ and $a_{s}^{\prime}$ is the sth species' anisotropy ratio defined in $\mathcal{R}^{\prime}$

$$
a_{s}^{\prime}=\frac{m_{s}\left(v_{s}-v_{f}\right)^{2}+T_{s x}}{T_{s y}}-1 .
$$

Figures 3(a)-3(c) compare the exact solution of the Weibelfilamentation dispersion relation (plain line) with the approximate expression (7) (dashed line) for three distinct plasma systems, comprising hot and cold ion populations in a neutralizing electron background. Overall, fairly good agreement is obtained, showing that the wave number that maximizes the growth rate verifies $k_{\mathrm{sat}} \simeq 0.5 k_{\max }$, where $k_{\max }$

$$
k_{\max }=\sqrt{\frac{\omega_{p c}^{2}}{c^{2}} a_{c}^{\prime}+\frac{\omega_{p h}^{2}}{c^{2}} a_{h}^{\prime}+\frac{\omega_{p e}^{2}}{c^{2}} a_{e}^{\prime}},
$$

is the non-vanishing wave number for which $\Gamma_{k_{y}}=0$.

In order to self-consistently evaluate the velocity of $\mathcal{R}^{\prime}$, one should take into account the variations of the plasma parameters involved in Eq. (6). For the sake of tractability, we only consider two interesting limits on $v_{f}$. When $v_{c} / v_{t h, c}^{2}$ $\gg v_{h} / v_{t h, h}^{2}$ or $T_{e} \ll T_{c}$, the $\mathcal{R}^{\prime}$ frame velocity can be approximated to $v_{f} \simeq v_{c}$. In the case of a forming Weibel-mediated shock, this limit is found to be valid only at the edge of the overlap region, far from the shock front [see Fig. 4(d)]. In this (a)

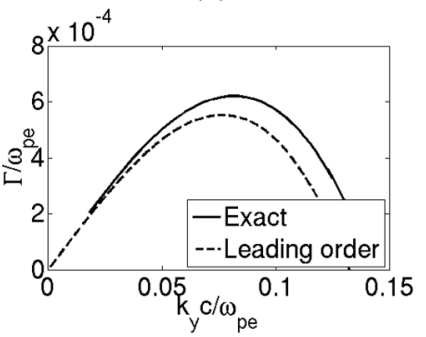

(b)

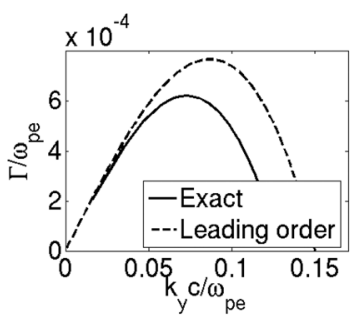

(c)

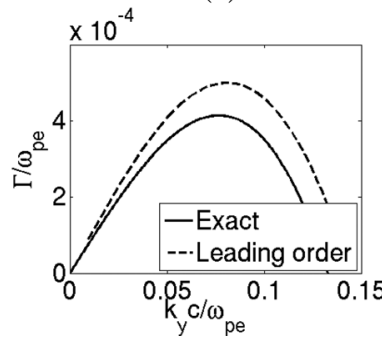

FIG. 3. Comparison of the exact Weibel growth rate (solid lines) with Eq. (7) (dashed lines). (a) $m_{i}=25 m_{e}, v_{c}=-v_{h}=0.4 c, v_{e}=0.36 c, T_{i h}=2 m_{e} c^{2}$. (b) $m_{i}=25 m_{e}, v_{c}=-v_{h}=0.2 c, v_{e}=0.18 c, T_{i h}=0.5 m_{e} c^{2}$. (c) $m_{i}=100 m_{e}, v_{c}=-v_{h}=0.4 c, v_{e}=0.36 c, T_{i h}=8 m_{e} c^{2}$. For the three cases, the population densities verifies $n_{h}=0.05, n_{c}=0.5, n_{e}=0.55, T_{e}=0.5 m_{e} c^{2}$, and $T_{i c}=0.05 m_{e} c^{2}$. The mean electron velocity is chosen to ensure global current neutralization. 
(a)

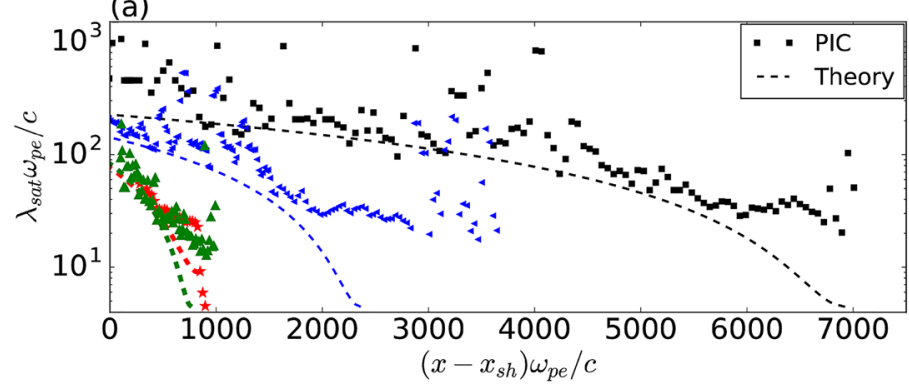

$$
\begin{aligned}
& \text { - } M_{i}=400, v_{0}=0.4 c \\
& \triangleleft \triangleleft M_{i}=100, v_{0}=0.4 c \\
& \star \star M_{i}=25, v_{0}=0.2 c \\
& \triangle \Delta M_{i}=25, v_{0}=0.4 c
\end{aligned}
$$
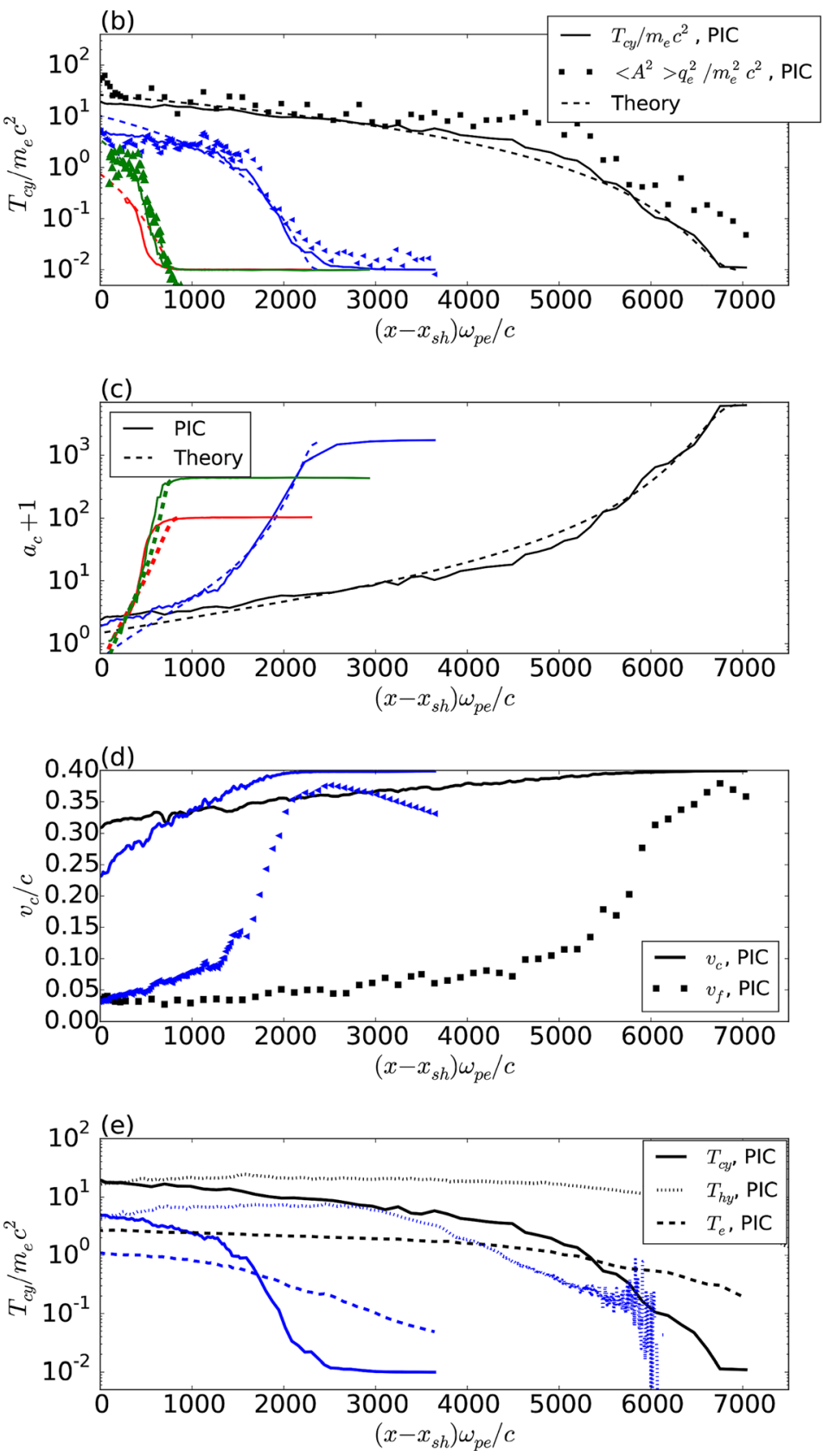

FIG. 4. Profiles of $\lambda_{\text {sat }} \omega_{p e} / c$ (a), $T_{c y} / m_{e} c^{2}$ (b), and $a_{c}+1$ (c) as a function of the distance (in units of $c / \omega_{p e}$ ) to the shock front, as extracted from four different simulations at times close to shock formation: $M_{i}=25, v_{i}=0.2 c$ (red stars, $\left.t \omega_{p e}=4200\right), M_{i}=25, v_{i}=0.4 c$ (green up-triangle, $\left.t \omega_{p e}=2100\right), M_{i}=100, v_{i}=0.4 c$ (blue lefttriangle, $\left.t \omega_{p e}=8650\right), M_{i}=400, v_{i}=0.4 c$ (black squares, $\left.t \omega_{p e}=17310\right)$. Predictions from Eqs. (23) $-(26)$ and $q_{e}^{2}\left\langle A_{x}^{2}\right\rangle \sim m_{e} T_{c y}$ are plotted in dashed lines (see text). Comparison between $T_{c y} / m_{e} c^{2}$ (plain lines) and $\left\langle A_{x}^{2}\right\rangle q_{e}^{2} / m_{e}$ (points) extracted from the PIC simulations are superimposed in panel (b). (d) Profile of $v_{c}$ extracted from the simulations. The calculation of $v_{f}$ using Eq. (6) and the various parameters measured in the simulations is plotted as dots in the case $M_{i}=400$ and $M_{i}=100$ for $v_{0}=0.4 c$. (e) Profiles of $T_{c y}, T_{h y}$, and $T_{e}$ extracted from the simulations. 
region, combining Eqs. (8) and (9) with $v_{f} \sim v_{c}$ shows that the dominant (fastest-growing) wavelength depends on the electron properties

$$
\frac{\lambda_{\mathrm{sat}} \omega_{p e}}{c} \sim 2 \pi \sqrt{\frac{T_{e}}{m_{e} v_{c}^{2}}} .
$$

Closer to the shock front, we may simplify Eq. (6) by applying current neutrality to the three populations, $n_{c} v_{c}$ $+n_{h} v_{h}+n_{e} v_{e}=0$. We thus obtain

$$
v_{f} \simeq \frac{\left(\frac{Z_{c}^{2}}{T_{c y}}-\frac{1}{T_{e y}}\right) n_{c} v_{c}+\left(\frac{Z_{h}^{2}}{T_{h y}}-\frac{1}{T_{e y}}\right) n_{h} v_{h}}{\frac{Z_{c}^{2} n_{c}}{T_{c y}}+\frac{Z_{h}^{2} n_{h}}{T_{h y}}+\frac{n_{e}}{T_{e y}}} .
$$

From Fig. 4(e), it appears that the electron (dashed lines) and ion (plain, cold and dotted, hot) temperatures verify $T_{e}$ $\ll T_{c}$ and $T_{e} \ll T_{h}$. For $Z_{h}=Z_{c}=1$, the above equation yields

$$
\left|v_{f}\right|<\frac{\left|n_{c} v_{c}+n_{h} v_{h}\right|}{n_{c}+n_{h}}<\left|v_{c}\right|
$$

so that the $\mathcal{R}^{\prime}$ frame velocity is smaller than the total ion mean velocity, which is, in turn, smaller than the cold-ion drift velocity. Finally, Fig. 4(d) supports the assumption $v_{f}$ $\ll v_{c}$, so that $\mathcal{R}^{\prime}$ can be approximately identified with the lab frame.

Consequently, $\lambda_{\text {sat }}$ verifies

$$
\frac{\lambda_{\mathrm{sat}} \omega_{p i}}{c} \simeq 4 \pi \sqrt{\frac{2 T_{c y}}{m_{i} v_{c}^{2}}}
$$

where use will be made of $n_{c} \simeq n_{h}$ and $Z_{c}=Z_{h}=Z_{i}$ in the following numerical applications. The two unknown plasma parameters involved in the above equation are the cold ion velocity $v_{c}$ and transverse temperature $T_{c y}$.

Our simulations indicate that, due to significant electron magnetization, the magnetic-field strength remains approximately constant over the nonlinear phase. The total current density carried, on average, by one ion filament, $j_{x} \simeq \kappa j_{c}$, involves the (cold) ion current contribution, $j_{c} \simeq q_{e} Z_{i} n_{i} v_{c}$ and the electron screening factor $\kappa(t) \simeq 4 c / \lambda_{\text {sat }}(t) \omega_{p e}{ }^{33}$ The associated magnetic field strength and potential vector can therefore be estimated by ${ }^{26}$

$$
\begin{gathered}
B_{z} \sim v_{c}\left(\mu_{0} m_{e} Z_{i} n_{i}\right)^{1 / 2}, \\
A_{x} \sim \frac{\lambda_{\mathrm{sat}} B_{z}}{2 \pi} \sim \frac{\lambda_{\mathrm{sat}} v_{c}}{2 \pi}\left(\mu_{0} m_{e} Z_{i} n_{i}\right)^{1 / 2},
\end{gathered}
$$

where $\mu_{0}$ is the vacuum permittivity.

Making use of Eq. (13), we then obtain

$$
m_{e} T_{c y} \sim Z_{i} q_{e}^{2}\left\langle A_{x}^{2}\right\rangle .
$$

Figure 4(b) shows very good agreement between $q_{e}^{2}\left\langle A_{x}^{2}\right\rangle$ and $m_{e} T_{c y}$ for three different shock simulations with $Z_{i}=1$ (plain lines and squares). Plugging Eq. (16) into Eq. (13) allows us to express the dominant wave number as a function of $q_{e}^{2}\left\langle A_{x}^{2}\right\rangle$

$$
\frac{Z_{i} q_{e}^{2}}{m_{e}}\left\langle A_{x}^{2}\right\rangle \sim T_{c y} \simeq \frac{m_{i} v_{c}^{2}}{2} \frac{\omega_{p i}^{2} \lambda_{\mathrm{sat}}^{2}}{16 \pi^{2} c^{2}}
$$

\section{B. Upstream filament dynamics}

The coalescence dynamics of the filaments formed by the incoming ions upstream of the shock can be modeled as in Ref. 25. At a fixed position in the downstream frame, the following energy conservation law approximately applies:

$$
d\left[m_{i}\left(d_{t} \lambda_{\mathrm{sat}}\right)^{2}\right]=\frac{j}{n} d A
$$

where $n$ is the ion density and $A$ is the amplitude of the longitudinal potential vector, estimated as $A \sim \sqrt{2\left\langle A_{x}^{2}\right\rangle}$. Equations (13)-(18) can be approximately solved by assuming that, at the start time of the nonlinear Weibel phase $\left(t=t_{*}\right), \lambda_{\text {sat }}\left(t_{*}\right)=\lambda_{*}$ and $\partial_{t} \lambda_{\text {sat }}\left(t_{*}\right)=0$. Making use of Eq. (17) and approximating $n \sim 2 n_{c}$, one can recast Eq. (18) as

$$
\left[\left(\partial_{t} \lambda_{\mathrm{sat}}\right)^{2}\right]_{t_{*}}^{t}=\frac{Z_{i} m_{e}}{2 \pi m_{i}} \int_{\lambda_{*}}^{\lambda_{\text {sat }}} \frac{v_{c}}{\lambda} \partial_{\lambda}\left(v_{c} \lambda\right) d \lambda .
$$

For simplicity, $v_{c}$ will hereafter be assumed independent of space. This approximation, which enables analytical progress, holds only in the region where the magnetic fields have not slowed down significantly the ions. To assess the implications of this approximation, one may apply conservation of energy to the incoming ion beam, $d\left[T_{c y}+T_{c x}+m_{i} v_{c}^{2}\right]=0$. Using $T_{c x}$ $\simeq T_{c y}$ yields $\delta T_{c y}=-m_{i} v_{c} \delta v_{c}$, and hence

$$
\frac{\delta T_{c y}}{T_{c y}}=-\frac{m_{i} v_{c} \delta v_{c}}{T_{c y}} \sim-a_{c}(0) \frac{\delta v_{c}}{v_{c}},
$$

where the initial ion anisotropy ratio is estimated to be $a_{c}(0) \sim m_{i} v_{c}^{2} / T_{c y}$. This equation therefore shows that, provided $a_{c}(0)$ is large enough, the system may simultaneously verify $\left|\delta v_{c} / v_{c}\right| \ll 1$ and $\left|\delta T_{c y} / T_{c y}\right| \gg 1$. For the simulations run with $\left(m_{i} / m_{e}, v_{0} / c\right)=(25,0.4),(100,0.4)$, and $(400,0.4)$, we thus find that a variation of $\delta v_{c} / v_{c} \sim-0.2$ corresponds to $\delta T_{c y} / T_{c y} \sim 16,64$, and 260, respectively. Hence, the larger is the initial ion anisotropy, the wider is the range of validity of the following approximate solution.

Neglecting the variations of $v_{c}$, Eq. (19) thus gives

$$
\partial_{t} \lambda_{\mathrm{sat}} \simeq v_{c} \sqrt{\frac{Z_{i} m_{e}}{2 \pi m_{i}} \ln \left(\frac{\lambda_{\mathrm{sat}}}{\lambda_{*}}\right)} .
$$

There results

$$
\Delta t v_{c} \sqrt{\frac{Z_{i} m_{e}}{2 \pi m_{i}}}=\int_{\lambda_{*}}^{\lambda_{\text {sat }}} \frac{d \lambda}{\sqrt{\ln \left(\lambda / \lambda_{*}\right)}}=\sqrt{\pi} \lambda_{*}\left[\operatorname{erfi}\left(\sqrt{\ln \left(\frac{\lambda_{\text {sat }}}{\lambda_{*}}\right)}\right)\right],
$$

where erfi is the complex error function ${ }^{34}$ and $\Delta t=t-t_{*}$. The dominant wavelength therefore reads 


$$
\lambda_{\text {sat }}=\lambda_{*} \exp \left[\operatorname{erfi}^{-1}\left(\frac{\Delta t}{\tau}\right)^{2}\right] \equiv \lambda_{*} F\left(\frac{\Delta t}{\tau}\right)
$$

where, besides the function $F$, we have introduced the effective timescale for the magnetic wavelength variation

$$
\tau=\pi \frac{\lambda_{*}}{v_{c}} \sqrt{\frac{2 m_{i}}{Z_{i} m_{e}}} .
$$

Equation (23) predicts that the dominant wavelength increases in time as $\lambda_{\text {sat }} / \log \left(\lambda_{\text {sat }}\right) \propto t$, i.e., at a rate slower than obtained in the symmetric configuration, $\lambda_{\text {sat }} \simeq \lambda_{*}\left(1+\Delta t^{2} / \tau_{0}^{2}\right)$. ${ }^{25}$ The value of $q_{e}^{2}\left\langle A_{x}^{2}\right\rangle$ can be obtained by combining Eqs. (17) and (23), yielding

$$
\frac{Z_{i} q_{e}^{2}}{m_{e}}\left\langle A_{x}^{2}\right\rangle \simeq T_{c y}(t) \simeq \frac{m_{i} v_{c}^{2}}{2} \frac{\omega_{p i}^{2} \lambda_{*}^{2} F(\Delta t / \tau)^{2}}{16 \pi^{2} c^{2}}
$$

Assuming that $m_{i} v_{c}^{2}+T_{c x}+T_{c y}$ is roughly constant, and approximating $T_{c x} \simeq T_{c y}$, we can infer the ion anisotropy

$$
a_{c}(t) \simeq \frac{m_{i} v_{c}^{2}\left(t_{*}\right)+T_{c x}\left(t_{*}\right)+T_{c y}\left(t_{*}\right)}{T_{c y}(t)}-2,
$$

where $T_{c y}$ is given by Eq. (25).

\section{PROFILE OF THE SHOCK TRANSITION REGION}

\section{A. Analytical formula of the dominant magnetic wavelength}

Equations (17) and (23) explicitly describe the temporal evolution of the field parameters at a given position in the upstream region, assuming that the Weibel instability has entered its nonlinear phase at time $t_{*}$. In order to obtain the spatial field profile, we make use of the shock front position, defined by Eq. (1), and of the dominant wavelength at the shock front $\lambda_{\text {front }} \equiv \lambda_{\text {sat }}\left(t_{\text {iso }}\right)$. Assuming $\lambda_{\text {front }}$ and $t_{\text {front }}$ are known, the profile of $\lambda_{\text {sat }}$ can be recast independent of $t_{*}$

$$
\frac{\lambda_{\text {sat }}}{\lambda_{*}}=F\left[\frac{t-t_{\text {iso }}}{\tau}-\frac{\left|x-x_{0}\right|}{\tau v_{\text {sh }}}-F^{-1}\left(\frac{\lambda_{\text {front }}}{\lambda_{*}}\right)\right],
$$

where $t-t_{\text {front }}>0, \lambda_{*} \leq \lambda_{\text {sat }} \leq \lambda_{\text {front }}$ and $F^{-1}(x)=$ erfi $[\sqrt{\ln (x)}]$ is the inverse function of $F$. The dominant magnetic wavelength at the shock front, $\lambda_{\text {front }}$, can be estimated by expressing ion isotropization, $T_{c y}\left(t_{\text {front }}\right) \sim 0.5 m_{i} v_{c}^{2}$, in Eq. (13), which yields

$$
\lambda_{\text {front }}=\lambda_{\text {sat }}\left(t_{\text {iso }}\right) \simeq 4 \pi \frac{c}{\omega_{p i}},
$$

where $\omega_{p i}$ is the total ion plasma frequency in the overlap region. Note that plugging Eq. (28) into Eq. (17) gives $T_{c y} \simeq 0.5 m_{i} v_{i}^{2}$, which is precisely the ion temperature value expected after complete isotropization.

Our calculation assumes that, once formed, the shock presents a stationary profile, defined by $q_{e}^{2}\left\langle A_{x}^{2}\right\rangle$ and $\lambda_{\text {sat }}$, in a frame moving at $v_{\mathrm{sh}}$. In Eqs. (27) and (17), the sharpness of the profile depends on the factor $F^{-1}\left(\frac{\lambda_{\text {front }}}{\lambda_{*}}\right)=$ erfi $\left(\sqrt{\ln \left(\lambda_{\text {front }} / \lambda_{*}\right)}\right)$. The thickness of the magnetic ionscattering region can be estimated by identifying it with the length needed for $\lambda_{\text {sat }}$ to increase from $\lambda_{*}$ to $\lambda_{\text {front }}$. For a large ion mass, the thickness of the upstream turbulent layer required to quasi-isotropize the cold ion beam, $L_{\text {iso }}=v_{\text {sh }}\left(t_{\text {iso }}-t_{*}\right)$, writes

$$
\begin{aligned}
L_{\text {iso }} & =40 \frac{c}{\omega_{p i}} \operatorname{erfi}\left[\sqrt{\log \left(\sqrt{\frac{m_{i}}{Z_{i} m_{e}}}\right)}\right], \\
& \simeq 35 \frac{c}{\omega_{p i}}\left(\frac{m_{i}}{Z_{i} m_{e}}\right)^{0.4} \equiv 35 \frac{c}{\omega_{p i}} M_{i}^{0.4},
\end{aligned}
$$

where use has been made of $M_{i}=m_{i} / Z_{i} m_{e}$ and of

$$
\lambda_{*}=\lambda_{\mathrm{sat}}\left(t_{*}\right) \simeq 4 \pi c / \omega_{p e},
$$

obtained by substituting $T_{c y}\left(t_{*}\right) \sim m_{e} v_{c}^{2}$ into Eq. (13). Equation (30) represents an approximate fit of Eq. (29), valid in the range $10 \leq m_{i} / Z_{i} m_{e} \leq 4000 .^{26}$ As the variations of $v_{c}$ and, therefore, plasma compression are neglected, $L_{\text {iso }}$ does not precisely coincide with the length required to fulfill the $\mathrm{RH}$ relations, $L_{\mathrm{sh}}$. Our simulations suggest that, in a $2 \mathrm{D}$ geometry, $L_{\mathrm{sh}} \sim L_{\mathrm{iso}}$. However, since the $\mathrm{RH}$ relations predict a larger plasma compression in $3 \mathrm{D}$ than in $2 \mathrm{D}$, one may expect $L_{\text {sh }} \gtrsim L_{\text {iso }}$ in a realistic geometry.

Interestingly, Eq. (30) shows that the size of the upstream magnetic turbulent layer does not depend on the initial velocity of the plasma slabs, in agreement with the simulation results of Ref. 1. Moreover, this length, which depends on both the ion and electron masses, can be related to the isotropization time involved in Eq. (1) through $L_{\text {iso }} \simeq v_{0} t_{\text {iso. }}$.

We now compare our analytical predictions with the field and plasma profiles extracted from various simulations at times close to shock formation. In Figs. 4(a)-4(c) are plotted the simulated profiles of $\lambda_{\text {sat }}, q_{e}^{2}\left\langle A_{x}^{2}\right\rangle / m_{e} \simeq T_{c y}$ and $a_{i}$ obtained for $\left(v_{0} / c, m_{i} / m_{e}\right)=(0.4,400),(0.2,100),(0.4,100)$, and $(0.4,25)$. Good quantitative agreement is observed over most of the (upstream) profiles, although the dominant magnetic wavelength seems to be somewhat underestimated far from the shock front. This discrepancy can be explained by noting that in Fig. 4(e), for $\left|x-x_{\mathrm{sh}}\right| \omega_{p e} / c>6000$ for $M_{i}=400$ (black), and for $\left|x-x_{\mathrm{sh}}\right| \omega_{p e} / c>2000$ for $M_{i}=100$ (blue), the assumption $T_{e} \ll T_{c}$ made in simplifying the dispersion relation is not valid. In these regions, however, the temperature and wavelength profiles are consistent with Eq. (10), derived for $T_{e} \gg T_{c}$, and which gives here $\lambda_{\text {sat }} \gtrsim 10 c / \omega_{p e}$ for $T_{e} \gtrsim 0.2 m_{e} c^{2}$ [see Fig. 4(e)].

Overall, the model predictions for the ion temperature and anisotropy are in good agreement with the simulated profiles over the whole shock transition regions. This demonstrates that our model correctly captures the field and plasma evolution, and, also, that Eq. (30) provides a good estimate of the shock transition length. The position of the shock front, as defined by Eq. (1), $a_{c}\left(x_{0}\right) \lesssim 1$ [see Fig. 4(c)], corresponds to the limit beyond which the magnetic spectrum ceases to be mainly transverse. Figure 1(a) indeed evidences 
that, in the region $3500 \lessgtr x \omega_{p e} / c \lessgtr 4000$ where $a_{c} \lessgtr 1$, the ion filaments have become close to isotropic, in contrast to the assumption of a purely transverse spectrum underlying our model [red dashed curve of Fig. 1(d)].

For $\left(M_{i}, v_{0} / c\right)=(25,0.4)$, a comparison with our estimates can be made once the shock fulfills the RH jump conditions. Figures 1(c) and 1(d) plot the cold ion anisotropy and temperature profiles at $t \omega_{p e}=4830$, i.e., when the shock front has propagated over a distance $\sim 750 \mathrm{c} / \omega_{\text {pe }}$. Fairly good agreement is obtained close to the shock front $\left(a_{c} \lesssim 1\right)$. Comparing the PIC and theoretical profiles close to [Figs. 4(b) and 4(d), green triangles], and after [Figs. 1 (c) and 1(d), black line] shock formation reveals that a discrepancy sets in around $x \omega_{p e} / c \lessgtr 3000$ and $x \omega_{p e} / c \gtrsim 5500$. This discrepancy could result from our somewhat coarse description of the plasma distribution, especially of the variations (in density and velocity) of the transmitted/reflected ions during shock propagation. Moreover, the strong filament deformations that can be seen in this region suggest that other mechanisms than coalescence could be of importance. Hence, our model is expected to hold during the early-time shock propagation, i.e., for $\left|t_{\text {front }}-t_{\text {iso }}\right| \lesssim t_{\text {iso }}$ where $t_{\text {front }}$ fulfills Eq. (1).

\section{B. Verification of the assumptions}

The present model relies on equations that are, in principle, only valid within a homogeneous system, which is formally not the case upstream of the shock. However, our theory may still hold in the inhomogeneous upstream region provided that $\lambda_{\text {sat }}$ varies faster than the incoming ion velocity. Consequently, for a small value of $d_{t} \lambda_{s a t} / v_{c}$, the spatial gradients should be negligible and hence, the local equations used in the model should remain valid. Making use of Eq. (21), we can show that

$$
\frac{d_{t} \lambda_{\text {sat }}}{v_{c}} \simeq 0.4 \frac{v_{0}}{v_{c}(t)} \sqrt{\frac{Z_{i} m_{e}}{m_{i}} \log \left(\frac{\lambda_{\mathrm{sat}}(t)}{\lambda_{*}}\right)},
$$

which gives $d_{t} \lambda_{s a t} / v_{c} \leqq 0.76 \sqrt{Z_{i} m_{e} / m_{i}} v_{0} / v_{c}$, much smaller than unity for $M_{i} \gg 1$, even at the shock front where $v_{c}$ does not vanish, as observed numerically [black plain lines in Fig. 2(b) and plain lines in Fig. 4(d)]. We have also assumed $v_{f} \simeq 0$ [Eq. (6)] in order to derive an approximate solution of the dispersion relation. Figure 4(d) shows the estimates of $v_{f}$ computed in two of our simulations, showing that $v_{f} / v_{0} \ll 1$ is well verified except in a region localized far from the shock front.

\section{CONCLUSIONS AND PROSPECTS}

In summary, we have investigated the field and plasma evolution upstream of Weibel-mediated collisionless shock fulfilling the Rankine-Hugoniot conditions. The stability properties of the upstream plasma have been analyzed both numerically and analytically, through approximate expressions of the dominant unstable modes. We have demonstrated that the instability is triggered by the overlap of the cold incoming ions, propagating towards the shock, and the outcoming hot ions, generated at the shock front and propagating in the opposite direction. We have then obtained a simple relation between the field quantity $q_{e}^{2}\left\langle A_{x}^{2}\right\rangle$ and the dominant wave number, valid in the upstream region. This relation has been combined with the simple coalescence model proposed in Ref. 25, giving analytical expressions of the magnetic and plasma profiles. The model predictions have been successfully compared with large-scale PIC simulations in the parameter range $v_{0} \in(0.2 c, 0.4 c)$ and $m_{i} / m_{e}$ $\in(25,100,400)$. Our model also predicts the thickness of the upstream turbulent region over which the ions are scattered up to quasi-isotropization. The expression obtained only depends on the ion and electron masses, independent of the other plasma properties and/or time history of the system. However, far from the shock front, the various instabilities developing prior to the ion Weibel filamentation may heat the ions and electrons, and thus smooth out the temperature profiles, so that our predictions may fail at times $\gg L_{\text {iso }} / v_{0}$.

In order to make the model tractable, we have neglected the variations of the incoming ion drift velocity, an assumption justified for large ion-to-electron mass ratios and up to a region close to the shock front, where the ion beam anisotropy gets close to unity. Inside the downstream region, as one expects the ions to be totally isotropized, this assumption no longer holds and so our predictions cease to be valid.

To conclude, this work provides the first predictive model of the transition region of a Weibel-mediated electron-ion shock wave. Yet, substantial further analysis is required for a complete understanding of the shock structure, explicitly taking account of particle scattering and alternate decay processes (e.g., kinklike modes ${ }^{35}$ ) for the magnetic filaments across the shock front, where they evolve into isotropic clumpy structures. The nonlinear damping of the latter structures in the downstream region also deserves further investigation as it is expected to influence the radiative properties of the shock. ${ }^{30,36}$ Finally, in this model, the contribution of the electrons to the shock formation is restricted to their screening the ion micro-currents. This simplification prevents us from describing the energy partition dynamics of a collisionless shock wave.

\section{ACKNOWLEDGMENTS}

The authors gratefully acknowledge F. Amiranoff, M. E. Dieckmann, F. Fiuza, M. Grech, M. Lemoine, M. Lobet, J. M. Rax, and V. T. Tikhonchuk for insightful discussions. The PIC simulations were performed using HPC resources at TGCC/CCRT. We acknowledge PRACE for awarding us access to TGCC/Curie (Grant No. 2014112576). C.R. acknowledges support from Grant No. ANR-11-IDEX-000402 Plas@Par. L.G. acknowledges support from the French National Agency for Research (ANR) through the project ANR-14-CE33-0019 MACH.

\footnotetext{
${ }^{1}$ T. N. Kato and H. Takabe, Astrophys. J., Lett. 681, L93 (2008).

${ }^{2}$ A. Spitkovsky, Astrophys. J., Lett. 682, L5 (2008).

${ }^{3}$ S. F. Martins, R. A. Fonseca, L. O. Silva, and W. B. Mori, Astrophys. J., Lett. 695, L189 (2009).

${ }^{4}$ L. Sironi and A. Spitkovsky, Astrophys. J. 726, 75 (2011).
} 
${ }^{5}$ M. E. Dieckmann, A. Meli, P. K. Shukla, L. O. C. Drury, and A. Mastichiadis, Plasma Phys. Control. Fusion 50, 065020 (2008).

${ }^{6}$ J. Niemiec, M. Pohl, A. Bret, and V. Wieland, Astrophys. J. 759, 73 (2012).

${ }^{7}$ A. Stockem, F. Fiuza, A. Bret, R. A. Fonseca, and L. O. Silva, Sci. Rep. 4, 3934 (2014).

${ }^{8}$ A. Stockem, T. Grismpayer, R. A. Fonseca, and L. O. Silva, Phys. Rev. Lett. 113, 105002 (2014).

${ }^{9}$ R. D. Blandford and J. P. Ostriker, Astrophys. J. 221, L29 (1978).

${ }^{10}$ F. C. Jones and D. C. Ellison, Space Sci. Rev. 58, 259 (1991).

${ }^{11}$ T. Piran, Rev. Mod. Phys. 76, 1143 (2005).

${ }^{12}$ A. Bret, L. Gremillet, and M. E. Dieckmann, Phys. Plasmas 17, 120501 (2010).

${ }^{13}$ Y. Kuramitsu, Y. Sakawa, T. Morita, C. D. Gregory, J. N. Waugh, S. Dono, H. Aoki, H. Tanji, M. Koenig, N. Woolsey, and H. Takabe, Phys. Rev. Lett. 106, 175002 (2011).

${ }^{14}$ W. Fox, G. Fiksel, A. Bhattacharjee, P.-Y. Chang, K. Germaschewski, S. X. Hu, and P. M. Nilson, Phys. Rev. Lett. 111, 225002 (2013).

${ }^{15}$ J. S. Ross, H.-S. Park, R. Berger, L. Divol, N. L. Kugland, W. Rozmus, D. Ryutov, and S. H. Glenzer, Phys. Rev. Lett. 110, 145005 (2013).

${ }^{16}$ C. M. Huntington, F. Fiuza, J. S. Ross, A. B. Zylstra, R. P. Drake, D. H. Froula, G. Gregori, N. L. Kugland, C. C. Kuranz, M. C. Levy, C. K. Li, J. Meinecke, T. Morita, R. Petrasso, C. Plechaty, B. A. Remington, D. D. Ryutov, Y. Sakawa, A. Spitkovsky, H. Takabe, and H. S. Park, Nat. Phys. 11, 173 (2015).

${ }^{17}$ A. J. Mackinnon, P. K. Patel, R. P. Town, M. J. Edwards, T. Phillips, S. C. Lerner, D. Price, D. Hicks, M. H. Key, and S. Hatchett, Rev. Sci. Instrum. 75, 3531 (2004).

${ }^{18}$ N. L. Kugland, D. D. Ryutov, C. Plechaty, J. S. Ross, and H.-S. Park, Rev. Sci. Instrum. 83, 101301 (2012).

${ }^{19}$ N. Kugland, D. D. Ryutov, P.-Y. Chang, R. P. Drake, G. Fiksel, D. H. Froula, S. H. Glenzer, G. Gregori, M. Grosskopf, M. Koenig, Y. Kuramitsu, C. Kuranz, M. C. Levy, E. Liang, J. Meinecke, F. Miniati, T. Morita, A. Pelka, C. Plechaty, R. Presura, A. Ravasio, B. A. Remington, B. Reville, J. S. Ross, Y. Sakawa, A. Spitkovsky, H. Takabe, and H.-S. Park, Nat. Phys. 8, 809 (2012).
${ }^{20}$ D. D. Ryutov, F. Fiuza, C. M. Huntington, J. S. Ross, and H.-S. Park, Phys. Plasmas 21, 032701 (2014).

${ }^{21}$ H.-S. Park, C. M. Huntington, F. Fiuza, R. P. Drake, D. H. Froula, G. Gregori, M. Koenig, N. L. Kugland, C. C. Kuranz, D. Q. Lamb, M. C. Levy, C. K. Li, J. Meinecke, T. Morita, R. D. Petrasso, B. B. Pollock, B. A. Remington, H. G. Rinderknecht, M. Rosenberg, J. S. Ross, D. D. Ryutov, Y. Sakawa, A. Spitkovsky, H. Takabe, D. P. Turnbull, P. Tzeferacos, S. V. Weber, and A. B. Zylstra, Phys. Plasmas 22, 056311 (2015).

${ }^{22}$ L. O. Drury, Rep. Prog. Phys. 46, 973 (1983).

${ }^{23}$ A. Bret, A. Stockem, F. Fiuza, C. Ruyer, L. Gremillet, R. Narayan, and L. O. Silva, Phys. Plasmas 20, 042102 (2013).

${ }^{24}$ A. Bret, A. Stockem, R. Narayan, and L. O. Silva, Phys. Plasmas 21, 072301 (2014).

${ }^{25}$ C. Ruyer, L. Gremillet, A. Debayle, and G. Bonnaud, Phys. Plasmas 22, 032102 (2015).

${ }^{26}$ C. Ruyer, L. Gremillet, G. Bonnaud, and C. Riconda, Phys. Rev. Lett. 117, 065001 (2016).

${ }^{27}$ A. S. Novo, A. Bret, R. A. Fonseca, and L. O. Silva, Astrophys. J., Lett. 803, L29 (2015).

${ }^{28}$ U. Keshet, B. Katz, A. Spitkovsky, and E. Waxman, Astrophys. J., Lett. 693, L127 (2009).

${ }^{29}$ E. Lefebvre, N. Cochet, S. Frizler, V. Malka, M.-M. Aléonard, J.-F. Chemin, S. Darbon, L. Disdier, J. Faure, A. Fedotoff, O. Landoas, G. Malka, V. Méot, P. Morel, M. Rabec Le Goahec, A. Rouyer, C. Rubbelynck, V. Tikhonchuk, R. Wrobel, P. Audebert, and C. Rousseaux, Nucl. Fusion 43, 629 (2003).

${ }^{30}$ P. Chang, A. Spitkovsky, and J. Arons, Astrophys. J. 674, 378 (2008).

${ }^{31}$ C. Ruyer, L. Gremillet, and G. Bonnaud, Phys. Plasmas 22, 082107 (2015).

${ }^{32}$ B. D. Fried, M. Gell-Mann, J. D. Jackson, and H. W. Wyld, J. Nucl. Energy, Part C 1, 190 (1960).

${ }^{33}$ A. Achterberg, J. Wiersma, and C. A. Norman, Astron. Astrophys. 475, 19 (2007).

${ }^{34}$ Handbook of Mathematical Functions with Formulas, Graphs and Mathematical Tables, edited by M. Abramowitz and I. A. Stegun (Dover, New York, 1964), pp. 126-140.

${ }^{35}$ M. Milosavljević and E. Nakar, Astrophys. J. 641, 978 (2006).

${ }^{36}$ M. Lemoine, J. Plasma Phys. 81, 455810101 (2015). 